\title{
Integração entre medidas de controle de enchentes e o aproveitamento social do espaço urbano na bacia do rio Jacaré, município do Rio de Janeiro
}

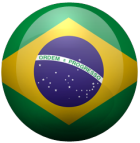 \\ Luana de Souza Bragança \\ Engenheira Ambiental, pesquisadora do Laboratório de Recursos Hídricos e Meio Ambiente (LabHidro) da \\ Universidade Federal Fluminense. Niterói [RJ] Brasil. <lbraganca@id.uff.br>

\section{Paulo Luiz da Fonseca} \\ Professor Adjunto do Departamento de Engenharia Civil, Universidade Federal Fluminense. Niterói [RJ] \\ Brasil. <paulofonseca@vm.uff.br>

\section{Monica de Aquino Galeano Massera da Hora} \\ Professora Adjunta do Departamento de Engenharia Agrícola e Meio Ambiente, Universidade Federal \\ Fluminense. Niterói [RJ] Brasil.<dahora@vm.uff.br>
}

\section{Resumo}

0 presente trabalho teve como objetivo propor uma abordagem sistêmica referente ao manejo de águas pluviais na bacia hidrográfica do rio Jacaré, localizado no Rio de Janeiro [RJ], contemplando um reservatório de detenção off-line aberto, com área total de $16.000 \mathrm{~m}^{2}$ e profundidade de $3 \mathrm{~m}$, operado por gravidade, para controle de enchentes na bacia. 0 hidrograma de projeto foi determinado pelo método do Soil Conservation Service (SCS), para tempos de recorrência de 5, 10 e 25 anos com duração crítica equivalente ao tempo de concentração. As vazões de pico obtidas, para os tempos de recorrência avaliados, foram de $38,78 \mathrm{~m}^{3} / \mathrm{s}$, $50,15 \mathrm{~m}^{3} / \mathrm{s}$ e $68,82 \mathrm{~m}^{3} / \mathrm{s}$, respectivamente. 0 routing do reservatório, obtido através do Método Modificado de Puls, resultou em amortecimento de $86 \%$ para um tempo de recorrência de 5 anos, $71 \%$ para 10 anos e $54 \%$ para 25 anos. 0 reservatório proposto foi idealizado de modo a permitir usos múltiplos e a integração com o espaço urbano, a partir da adequação da bacia de detenção em uma quadra de futebol de grama, além de uma área de vivência, um espaço de lazer para crianças e de exercícios para idosos. Desta forma, seria possível agregar valor social à comunidade local.

\section{Palavras-chave}

Bacia de detenção. Método Modificado de Puls. Aproveitamento social.

\section{Integration between flood control measures and the social enjoyment of the urban area in Jacaré river basin, Rio de Janeiro municipality}

\begin{abstract}
The present case study aimed to propose a systemic approach on the rainwater management in the Jacaré river watershed, located in Rio de Janeiro [RJ], contemplating an open off-line detention basin, with a total area of $16,000 \mathrm{~m}^{2}$ and a depth of $3 \mathrm{~m}$, operated through gravity, for flood control. The project hydrograph was determined by the Soil Conservation Service (SCS) method, for the return periods of 5, 10 and 25 years and critical time equivalent to the watershed's time of concentration. The peak flow obtained for the evaluated return periods was equivalent to $38.78 \mathrm{~m}^{3} / \mathrm{s}, 50.15 \mathrm{~m}^{3} / \mathrm{s}$ and $68.82 \mathrm{~m}^{3} / \mathrm{s}$, respectively. The reservoir routing obtained through Modified-Puls Routing resulted in a peak flow reduction of $86 \%$ for a 5-year return period, $71 \%$ for 10 years and $54 \%$ for 25 years. The proposed reservoir was designed to allow multiple uses and the integration with the urban space, through the adequacy of the detention basin on a grass soccer field, in addition to a living area, a leisure area for children and elderly exercises. Thus, it would be possible to add social value to the local community.
\end{abstract}

\section{Keywords}

Detention basin. Modified-Puls Routing, Social Use. 


\section{Introdução}

Os impactos gerados pela urbanização são cada vez mais evidentes e correlacionados. A expansão de áreas impermeáveis reduz a capacidade de infiltração do solo, diminuindo a recarga das águas subterrâneas. Como consequência, o escoamento superficial direto é intensificado e acelerado, contribuindo de forma significativa para as inundações urbanas em períodos de chuvas intensas. A alteração da topografia e geomorfologia da paisagem, causada pelo processo de urbanização, produz ainda efeitos diretos e indiretos sobre os rios, alterando seus regimes e volumes de cheias, devido principalmente à canalização e ocupação urbana inadequada das várzeas ribeirinhas (Tucci C. M., 2007; 2009; Seto \& Shepherd, 2009; Güneralp, Güneralp, \& Liu, 2015; Yang, Tian, \& Niyogi, 2015).

Outro relevante impacto da urbanização é o fenômeno da ilha de calor, que consiste em uma maior diferença entre as temperaturas, do ar e da superfície, das áreas urbanas e das áreas rurais em seu entorno. Este fenômeno gera um impacto na circulação atmosférica, alterando os padrões, intensidades, e distribuições espacial e temporal das precipitações, especialmente das convectivas que possuem grande intensidade em um curto período de tempo, agravando a problemática das enchentes urbanas. Isso porque a substituição de cobertura vegetal por materiais impermeáveis, reduz a taxa de evapotranspiração e umidade disponível, causando uma redução no fluxo de calor latente. Adicionalmente, devido ao baixo albedo destes materiais, que significa uma maior condutividade e capacidade térmica, ocorre um aumento no fluxo de calor sensível na região urbanizada. Estudos sugerem ainda, que emissões de calor antropogênico e a poluição atmosférica também influenciam a circulação atmosférica em áreas urbanas, intensificando a ilha de calor (Oke, 1987; Arnfield, 2003; Guo, Fu, \& Wang, 2006; Cheng \& Chan, 2012).

Além disso, a poluição causada pelos resíduos sólidos afeta o sistema de drenagem de águas pluviais urbana, uma vez que as águas transportam sedimentos e demais materiais ao escoar pelas superfícies. $\mathrm{O}$ assoreamento das calhas dos rios, ou estruturas de controle de enchente, pode agravar as inundações por reduzir a capacidade hidráulica destas, necessitando de procedimentos de dragagem para retirada do acúmulo de sedimentos depositados (Tucci C. M., 2007).

As inundações urbanas podem ainda acarretar doenças de veiculação hídrica. Em locais onde o esgoto sanitário é lançado nos corpos hídricos sem o tratamento adequado, ou onde é utilizado um sistema compartilhado para coleta de águas pluviais e de esgoto, a qualidade das águas pluviais é deteriorada e torna-se prejudicial à saúde humana e à natureza. Além disso, em locais onde exista carência de limpeza urbana adequada, a água das enchentes pode reagir com vetores e transmitir doenças, como a leptospirose (Tucci C. E., 2005; Taylor, et al., 2011; Kazmierczak \& Cavan, 2011).

O aumento da frequência e gravidade das enchentes urbanas, e a consequente pressão da população, vem gerando uma mudança na visão do planejamento urbano. A crescente necessidade de minimizar estes impactos, incentiva a implantação de medidas de prevenção e controle de enchentes, sendo a escolha destas dependente tanto do estágio de desenvolvimento da cidade, quanto da atuação desejada em relação ao hidrograma (Canholi, 2005; FCTH, 2012b).

As medidas estruturais, que constituem a construção de estruturas físicas, podem ser divididas em convencionais e não-convencionais. As primeiras referem-se ao manejo tradicional de águas pluviais através da canalização de rios e utilização de galerias, enquanto as últimas caracterizamse pela drenagem sustentável, englobando medidas de controle na fonte e à jusante, que visam a reintegração das águas pluviais com o meio ambiente, além da melhoria de sua qualidade, de forma a mitigar os impactos da urbanização (FCTH, 2012a; 2012b).

Existem ainda as medidas não-estruturais, que atuam como medidas preventivas relacionadas a programas de educação ambiental, melhoria dos sistemas de coleta de resíduos sólidos e de esgoto doméstico, investimento em estações e estudos de previsão meteorológica, além de sistemas de monitoramento e sirenes em encostas ocupadas (FCTH, 2012b).

O presente trabalho visa, portanto, abordar a temática do controle de enchentes urbanas em uma bacia hidrográfica da cidade do Rio de Janeiro, considerando aspectos técnico-financeiros e socioambientais. 


\section{Caracterização da Área de Estudo}

A bacia hidrográfica do Rio Jacaré, composta pelo Rio Jacaré e Rio Salgado, pertence à bacia do Canal do Cunha e está localizada na zona norte da cidade do Rio de Janeiro, conforme ilustra a Figura 1.

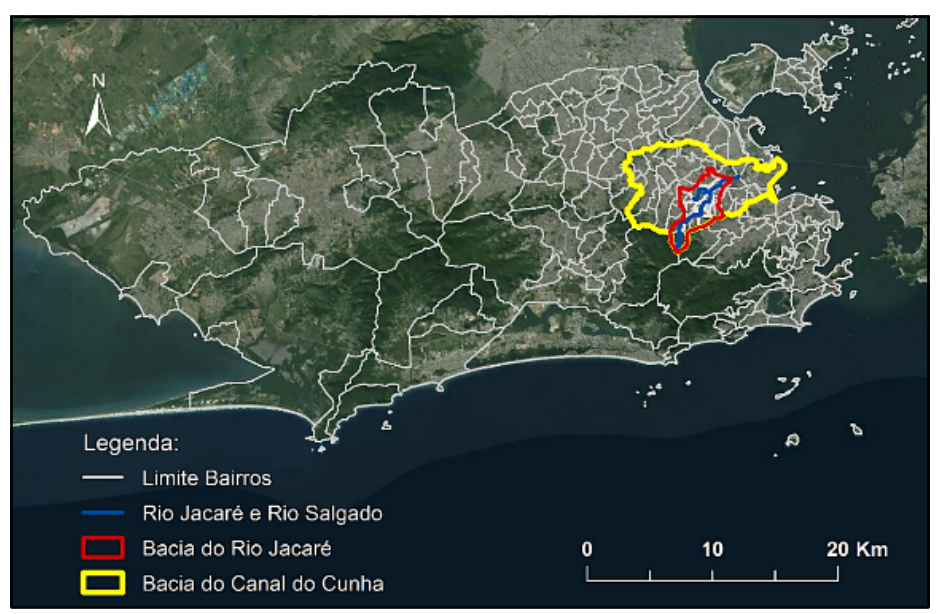

Figura 1. Localização da bacia hidrográfica do rio Jacaré na cidade do Rio de Janeiro [RJ].

O Rio Jacaré nasce no Maciço da Tijuca, mais precisamente na Serra do Mateus, a cerca de $630 \mathrm{~m}$ de altitude. De acordo com o Plano Diretor de Manejo de Águas Pluviais (PDMAP), da cidade do Rio de Janeiro para a Bacia do Canal do Cunha, elaborado pelo Consórcio Hidrostudio-FCTH (2013), o solo da bacia foi caracterizado como grupo hidrológico do tipo C, segundo critérios estabelecidos pela EMBRAPA (2004). A bacia possui uma área de aproximadamente $14,3 \mathrm{~km}^{2}$ com um relevo acentuado próximo a nascente do rio Jacaré, onde encontram-se encostas não urbanizáveis cobertas por Mata Atlântica. Esta topografia pode favorecer a ocorrência de processos erosivos próximo à cabeceira, devido à alta velocidade do escoamento superficial, e consequente assoreamento dos cursos d'água a jusante, reduzindo sua capacidade de escoamento e aumentando o risco de inundações.

A bacia estudada é predominantemente urbana e residencial, englobando algumas comunidades carentes. A maior parte do curso d'água está canalizado, com algumas seções cobertas, e próximo à sua foz encontra-se com seu leito natural. Segundo Hidrostudio-FCTH (2013), grande parte dos trechos dos rios estão assoreados, com capacidade hidráulica reduzida, conforme as Figura 2 e Figura 3.

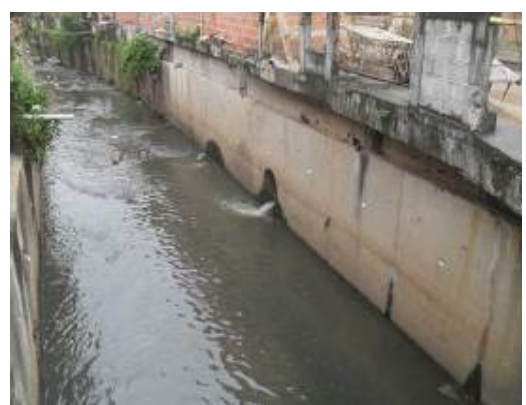

Figura 2. Trecho entre a Rua Dois de Maio e a Rua Lino Teixeira. Fonte: Hidrostudio-FCTH (2013)

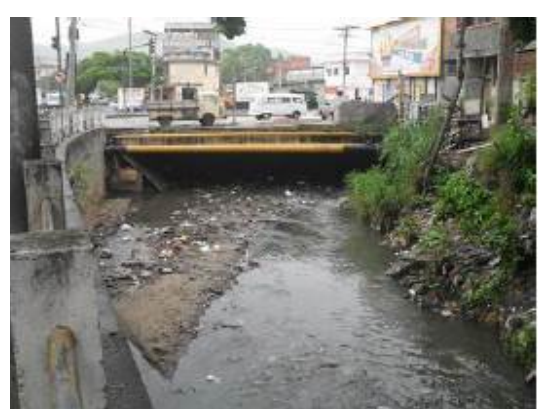

Figura 3. Travessia sob a Rua Lino Teixeira. Fonte: Hidrostudio-FCTH (2013)

Conforme o estudo de Bragança (2015), a bacia do Rio Jacaré, para uma chuva de 25 anos de recorrência, período recomendado pela instrução técnica da Fundação Rio Águas (2010) para projetos de drenagem urbana, teria um volume de escoamento superficial de $960.000 \mathrm{~m}^{3}$, com vazão máxima de $192 \mathrm{~m}^{3} / \mathrm{s}$. A avaliação hidráulica expedita dos trechos dos rios da bacia, feita neste estudo, mostrou que o sistema de drenagem seria incapaz de amortecer este escoamento, sendo necessárias medidas de controle de enchente para a região, corroborando com os resultados de Hidrostudio-FCTH (2013). 
O presente trabalho teve, portanto, o objetivo de propor um reservatório de detenção off-line aberto, com operação por gravidade, para controle de enchentes na bacia, visando usos múltiplos e a integração com o espaço urbano.

\section{Metodologia}

Os cálculos foram realizados com base nos dados de Hidrostudio-FCTH (2013), através de planilhas do Microsoft Excel, seguindo metodologias recomendadas. Os softwares AutoCad e ArcMap 10.3 foram utilizados para análise e geoprocessamento dos dados.

Os rios foram divididos em trechos conforme a homogeneidade das seções transversais, apresentadas por Hidrostudio-FCTH (2013). Através do software de geoprocessamento, as áreas da bacia foram separadas conforme o tipo de ocupação do solo, com base nas ortofotos em escala 1:25.000 disponibilizadas pelo IBGE (2014).

Os dados hidrológicos utilizados foram definidos conforme a área de influência das equações IDF dos postos pluviométricos Capela Mayrink e Sabóia Lima, através do método de Thiessen. Conforme explicado por Hidrostudio-FCTH (2013, p.20), apesar do Posto Benfica abranger a Bacia do Rio Jacaré, este apresenta coeficientes IDF muito destoantes de seus postos vizinhos, gerando menores valores de precipitação em relação à média destes. Devido a isso, este posto foi desconsiderado "a fim de se trabalhar a favor da segurança na estimativa das vazões de projeto", utilizando áreas de influência adaptadas para os postos vizinhos.

O local escolhido para construção do reservatório $R$ foi o terreno pertencente a antiga TeleRJ, localizado próximo à Rua Dois de Maio no bairro do Engenho Novo. Um dos fatores principais para a escolha do local foi sua localização a montante da confluência do Rio Jacaré com seu afluente, o Rio Salgado, uma vez que o volume de água transportado é maior à jusante desta e o reservatório controla enchentes à jusante de sua bacia (FCTH, 2012b).

A bacia do reservatório $R$ foi, portanto, determinada de acordo com a topografia da região, de modo a considerar toda a área que possui o reservatório como exutório do escoamento superficial, conforme apresenta a Figura 4.

As precipitações máximas foram calculadas pelo método dos blocos alternados, utilizando a Equação 1, juntamente com os coeficientes de Thiessen das áreas de influência dos postos pluviométricos.

$$
i=\frac{a \times T_{R}^{b}}{(t+c)^{d}}
$$

\section{Onde:}

$i=$ intensidade da chuva $(\mathrm{mm} / \mathrm{h})$;

$T_{R}=$ tempo de recorrência (anos);

$t=$ duração da chuva (min);

$a, b, c, d=$ coeficientes, conforme Tabela 1 .

Figura 4. Bacia do reservatório, localizado na bacia hidrográfica do rio Jacaré.

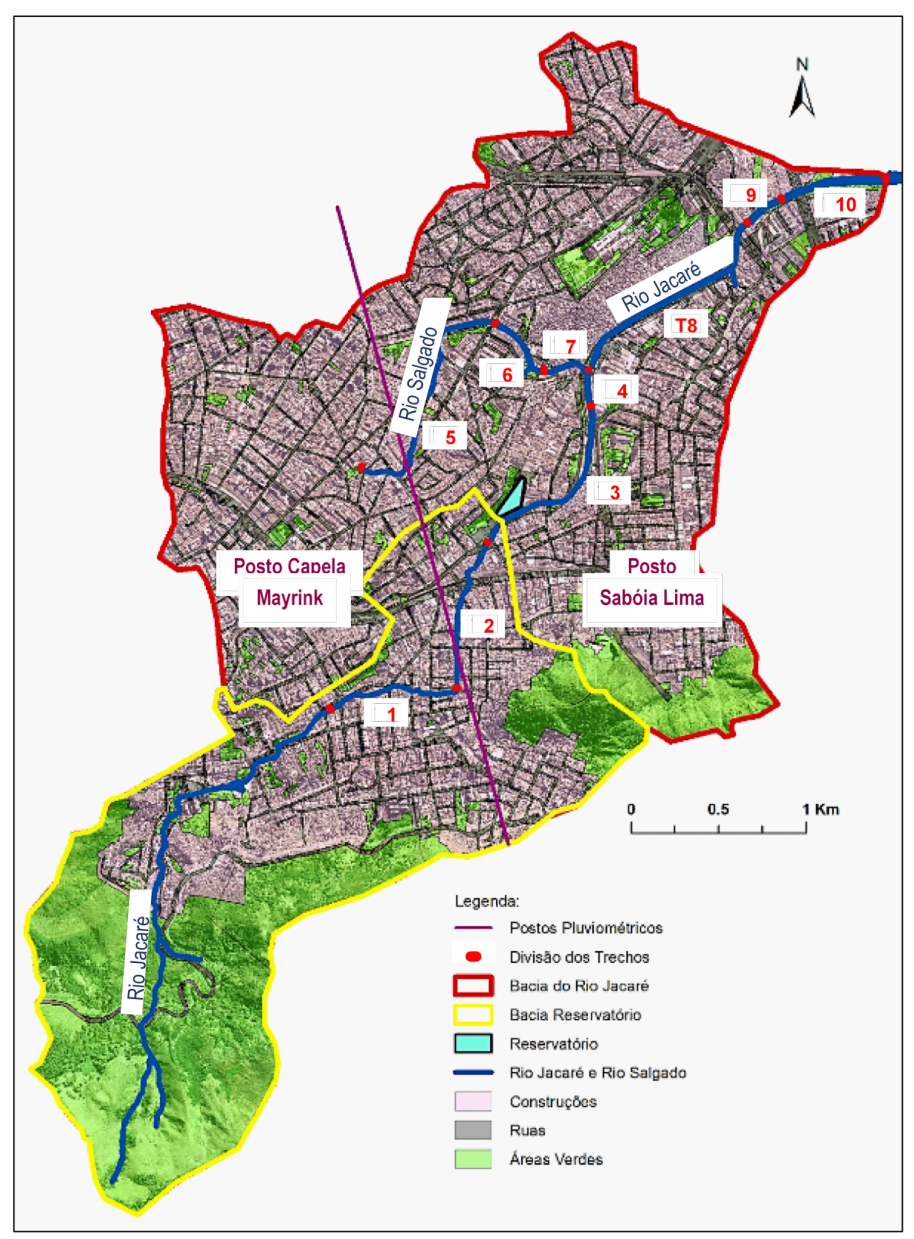

(C) Labor \& Engenho, Campinas [SP] Brasil, v.10, n.4, p.357-368, out./dez. 2016. 
Tabela 1. Coeficientes de chuvas IDF.

\begin{tabular}{|l|cccc|}
\hline Pluviômetro & a & b & c & d \\
\hline Capela Mayrink & 921,39 & 0,162 & 15,46 & 0,673 \\
\hline Sabóia Lima & $1.782,78$ & 0,178 & 16,60 & 0,841 \\
\hline
\end{tabular}

Fonte: Adaptado de Rio Águas, 2010.

O tempo de concentração nos trechos canalizados da bacia, foi calculado pelo método cinemático (Equação 2), e a velocidade através da fórmula de Manning (Equação 3) (Porto, 1995; Tomaz, 2002).

$$
\begin{gathered}
t_{c}=\frac{1}{60} \times \sum\left(l_{i} / V_{i}\right) \\
V=\frac{1}{n} \times R^{2 / 3} \times S^{0,5}
\end{gathered}
$$

Onde:

$t_{c}=$ tempo de concentração (min);

$L_{i}=$ comprimento de cada trecho homogêneo $(\mathrm{m})$;

$V_{i}=$ velocidade de cada trecho homogêneo $(\mathrm{m} / \mathrm{s})$;

$n=$ número de Manning;

$R=$ raio hidráulico $(\mathrm{m})$;

$S=$ declividade do canal $(\mathrm{m} / \mathrm{m})$.

Para estimar o tempo de concentração do percurso no terreno natural até o talvegue principal da bacia, foi utilizado o método de Kerby (Equação 4), considerando o tipo de superfície do terreno (Rio Águas, 2010).

$$
t_{c}=1,44 \times\left(L \times C_{R} / S^{0,5}\right)^{0,47}
$$

Onde:

$t_{c}=$ tempo de concentração (min);

$L=$ comprimento do percurso $(\mathrm{km})$;

$C_{k}=$ coeficiente referente ao tipo de superficie, utilizado: 0,4;

$S=$ declividade do terreno $(\mathrm{m})$.

0 trecho inicial do rio Jacaré foi extrapolado para montante até alcançar sua real extensão, uma vez que Hidrostudio-FCTH (2013) não forneceu informações completas do rio próximo a sua nascente. A velocidade máxima nos trechos foi considerada como 3,5 m/s, seguindo as considerações de Hidrostudio-FCTH (2013), do qual foram ainda retirados os dados referentes à bacia do reservatório $\mathrm{R}$, conforme a Tabela 2, e as

\begin{tabular}{|c|c|c|c|c|c|}
\hline Bacia & Posto Pluviométrico & $\begin{array}{c}\text { Coeficiente } \\
\text { de Thiessen }\end{array}$ & $A\left(\mathrm{~km}^{2}\right)$ & L (km) & $\mathbf{H}(\mathrm{m})$ \\
\hline $\mathrm{R}$ & $\begin{array}{l}\text { Capela Mayrink } \\
\text { Sabóia Lima }\end{array}$ & $\begin{array}{l}0,81 \\
0,19\end{array}$ & 5,700 & 5,113 & 710 \\
\hline
\end{tabular}
características dos trechos canalizados da bacia, apresentadas na Tabela 3.

Tabela 2. Dados da bacia do reservatório R.

Fonte: Adaptado de Hidrostudio-FCTH, 2013.

Tabela 3. Dados dos trechos do rio Jacaré pertencentes à bacia do reservatório $\mathrm{R}$.

\begin{tabular}{|ccccccccc|}
\hline Trecho & $\mathbf{y}(\mathbf{m})$ & $\mathbf{B}(\mathbf{m})$ & C.M. (m) & C.J. (m) & L (m) & S (m/m) & $\mathbf{n}$ \\
\hline 1 & 2,71 & 4,47 & 21,81 & 13,29 & 662,93 & 0,0129 & 0,017 \\
\hline 2 & 3,25 & 4,79 & 13,29 & 6,32 & 984,27 & 0,0071 & 0,017 \\
\hline $3^{*}$ & 3,73 & 7,26 & 6,32 & 5,24 & 162,08 & 0,0067 & 0,025 \\
\hline
\end{tabular}

* Foram consideradas as características do trecho 3, de seu início (junto ao trecho 2) até o reservatório R. Fonte: Adaptado de Hidrostudio-FCTH, 2013. 
Com estes cálculos iniciais, foi possível utilizar o modelo chuva-vazão desenvolvido pelo Soil Conservation Service (SCS) para determinar o hidrograma de projeto da bacia do reservatório. Para o cálculo do índice $\mathrm{CN}$ ponderado (Equação 5), foram utilizados os valores tabelados do método para os diferentes tipos de ocupação, considerando uma condição média de umidade antecedente do solo, para o grupo hidrológico C. A partir deste, foram calculadas a capacidade de armazenamento (Equação 6) e a precipitação excedente na bacia (Equação 7) (SCS, 1986; Canholi, 2005).

$$
\begin{gathered}
C N_{P O N D}=\sum_{i=1}^{n}\left(A_{i} \times C N_{i}\right) / A_{T} \\
S_{D}=\left(25400-254 \times C N_{P O N D}\right) / C N_{P O N D} \\
H_{E X C}=\left(P-I_{a}\right)^{2} /\left(P+0,8 \times S_{D}\right), \quad P>I_{a}
\end{gathered}
$$

Onde:

$S_{D}=$ armazenamento máximo $(\mathrm{mm})$;

$A_{i}=$ área da ocupação i $\left(\mathrm{km}^{2}\right)$;

$\mathrm{CN}_{i}=$ índice curva número correspondente à ocupação $i$;

$n=$ número total de tipos de ocupação;

$A_{T}=$ área total da bacia $\left(\mathrm{km}^{2}\right)$;

$H_{E X C}=$ precipitação excedente $(\mathrm{mm})$;

$P=$ precipitação $(\mathrm{mm})$;

$I_{a}=$ abstração inicial $(\mathrm{mm})$, considerada $20 \%$ do armazenamento máximo.

Em seguida, foram calculadas a vazão de pico (Equação 8) do hidrograma sintético resultante do método, o tempo de ocorrência desta vazão (Equação 9), o tempo de recessão (Equação 10) e o tempo de base do hidrograma (Equação 11), além da duração da chuva excedente unitária (Equação 12). Esta última foi utilizada como intervalo de tempo para determinação da precipitação efetiva e do hidrograma unitário (Porto, 1995; Canholi, 2005).

$$
\begin{gathered}
Q_{u, p}=0,208 \times A_{d} \div t_{p} \\
t_{p}=\frac{D_{u}}{2}+0,6 t_{c} \\
t_{r}=1,67 \times t_{p} \\
t_{B}=2,67 \times t_{p} \\
D_{U}=0,133 \times t_{c}
\end{gathered}
$$

Onde:

$Q_{u, p}=$ vazão de pico $\left(\mathrm{m}^{3} / \mathrm{s}\right)$;

$A_{d}=$ área de drenagem da bacia $\left(\mathrm{km}^{2}\right)$;

$t_{p}=$ tempo para ocorrência da vazão de pico (h);

$D_{U}=$ duração unitária da precipitação excedente $(h)$;

$t_{r}=$ tempo de recessão $(h)$;

$t_{B}=$ tempo de base (h);

$t_{c}=$ tempo de concentração $(h)$.

Após a determinação do hidrograma de projeto, foi utilizado o Método Modificado de Puls (Strelkoff, 1980) para os cálculos referentes ao routing do reservatório, seguindo a metodologia apresentada por Tomaz (2002).

0 reservatório proposto possui uma área de aproximadamente $16.000 \mathrm{~m}^{2} \mathrm{com} 3 \mathrm{~m}$ de profundidade, tendo uma capacidade de armazenamento total de $48.000 \mathrm{~m}^{3}$, conforme a Figura 5. Sua profundidade foi escolhida de modo a permitir seu funcionamento por gravidade, considerando os dados de Hidrostudio-FCTH (2013) referentes à cota de fundo do trecho do rio Jacaré a montante e a jusante do reservatório. 


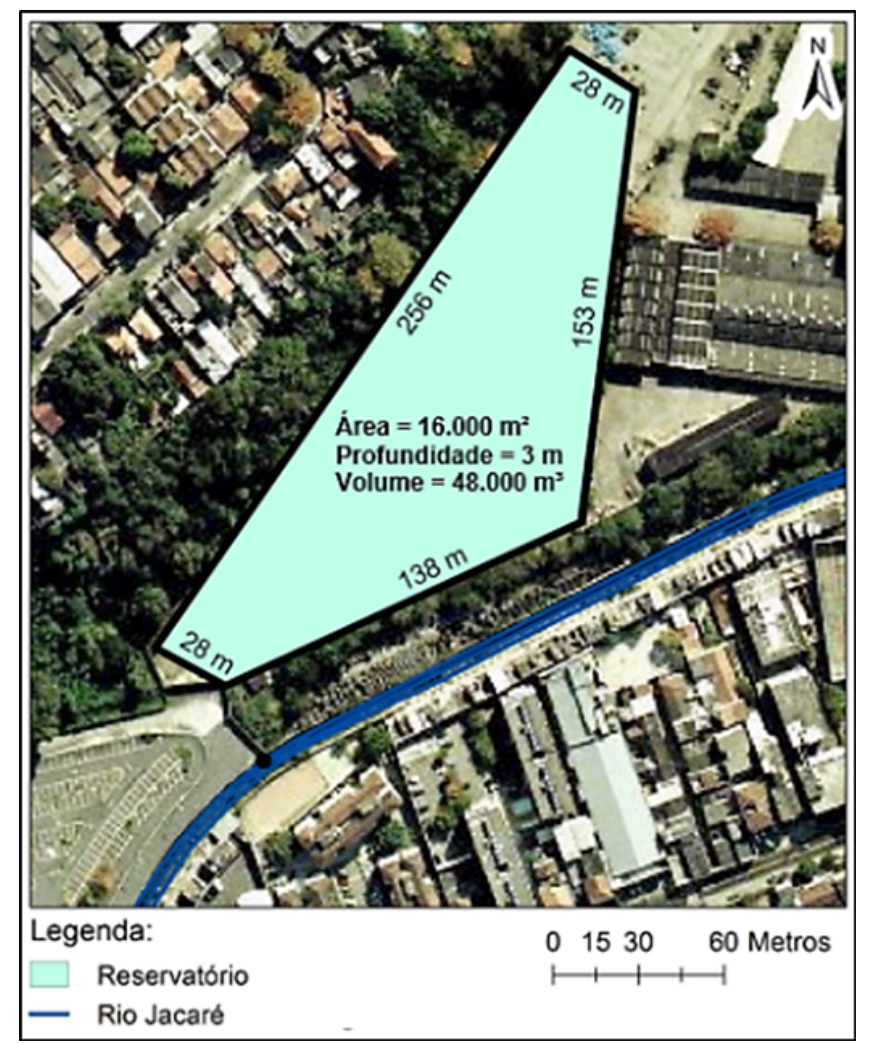

Figura 5. Localização e dimensões do reservatório R.
A equação inicial do routing de armazenamento (Equação 13), pode ser escrita na forma de diferenças finitas (Equação 14) (Tomaz, 2002; Canholi, 2005).

$$
\begin{gathered}
I-Q=\Delta S / \Delta t \\
\left(I_{1}+I_{2}\right)+\left(\frac{2 S_{1}}{\Delta t}-Q_{1}\right)=\left(\frac{2 S_{2}}{\Delta t}-Q_{2}\right)
\end{gathered}
$$

Onde:

$I=$ vazão afluente $\left(\mathrm{m}^{3} / \mathrm{s}\right) ;$

$Q=$ vazão defluente $\left(\mathrm{m}^{3} / \mathrm{s}\right)$;

$S=$ volume armazenado $\left(\mathrm{m}^{3}\right)$;

$t=$ tempo $(s)$;

$S_{1}$ e $S_{2}=$ volume armazenado no tempo 1 e $2\left(\mathrm{~m}^{3}\right)$; $Q_{1}$ e $Q_{2}=$ vazão defluente no tempo 1 e $2\left(\mathrm{~m}^{3} / \mathrm{s}\right)$.

$I_{1}$ e $I_{2}=$ vazão afluente no tempo 1 (tempo t) e no tempo 2 (tempo $t+\Delta t)\left(\mathrm{m}^{3} / \mathrm{s}\right)$;

Uma vez que os termos $I_{1}, I_{2}, Q_{1}$ e $S_{1}$ são conhecidos para qualquer tempo, e deseja-se obter os valores de $Q_{2}$, os valores de $S_{2}$ podem ser calculados a partir das relações das curvas cota $x$ volume e cota $x$ vazão defluente (Canholi, 2005). A primeira curva (Equação 15) foi calculada a partir dos coeficientes $b$ (Equação 16) e $c$ (Equação 17), definidos através de análise de regressão. Enquanto a segunda foi determinada pelas vazões de descarga final, através do somatório das vazões das estruturas de controle do reservatório, um vertedor retangular de soleira normal (Equação 18), e um orifício retangular (Equação 19) (Tomaz, 2002).

$$
S=b \times h^{c}
$$

$$
\begin{gathered}
C=\frac{\sum[(\log S) \times(\log h)]-\frac{\sum(\log S) \times \sum(\log h)}{N}}{\sum\left[(\log h)^{2}\right]-\left[\frac{\sum(\log h)}{N}\right]^{2}} \\
b=10^{\left[\sum(\log S)-c \times \sum(\log h)\right] / N} \\
Q_{w_{r e t}}=K_{w} \times L \times(2 g)^{0,5} \times h^{3 / 2} \\
Q_{0}=K_{0} \times A_{0} \times\left(2 g \times h_{0}\right)^{0,5}
\end{gathered}
$$

Onde:

$S=$ volume armazenado $(\mathrm{m})$;

$h=$ altura da água $(\mathrm{m})$;

$b=$ parâmetro $\left(m^{3-c}\right)$;

$c=$ parâmetro;

$N=$ número total de intervalos $\Delta h$;

$K_{w}=$ coeficiente tabelado, utilizado: 0,49;

$L=$ comprimento da crista do vertedor $(\mathrm{m})$;

$g=$ aceleração da gravidade $\left(\mathrm{m} / \mathrm{s}^{2}\right)$;

$h=$ altura da lâmina d'água sobre a crista $(\mathrm{m})$;

$Q_{o}=$ vazão de descarga do orifício $\left(\mathrm{m}^{3} / \mathrm{s}\right)$;

$K_{o}=$ coeficiente tabelado, utilizado: 0,49;

$Q_{w_{\text {ret }}}=$ vazão no vertedor retangular $\left(\mathrm{m}^{3} / \mathrm{s}\right)$;

$A o=$ área da seção transversal do orifício $\left(\mathrm{m}^{2}\right)$;

$h_{o}=$ altura da água sobre a geratriz superior do orifício (m). 
O procedimento de routing do Método Modificado de Puls foi feito pelas seguintes etapas (Akan, 1993):

1) Obtêm-se a curva de armazenamento $S$ em função da vazão de descarga total;

2) Seleciona-se um tempo $\Delta t$ e elabora-se um gráfico com abscissa $(2 S / \Delta t+Q)$ e ordenada igual a vazão de descarga total;

3) Calcula-se (I1+I2) para qualquer intervalo de tempo e $(2 \mathrm{~S} 1 / \Delta \mathrm{t}-\mathrm{Q} 1)$ da condição inicial ou tempo anterior;

4) Calcula-se $(2 \mathrm{~S} 2 / \Delta \mathrm{t}+\mathrm{Q} 2)$ através da soma de $(\mathrm{I} 1+\mathrm{I} 2) \operatorname{com}(2 \mathrm{~S} 1 / \Delta \mathrm{t}-\mathrm{Q} 1)$, conforme Equação 14;

5) Obtêm-se Q2 da relação feita na etapa 2, através de uma interpolação linear (Equação 20) com o valor calculado na etapa 4 ;

Onde:

$$
y=\frac{\left(x-x_{2}\right)}{\left(x_{1}-x_{2}\right)} * y_{1}+\frac{\left(x-x_{1}\right)}{\left(x_{2}-x_{1}\right)} * y_{2}
$$

$y=Q_{2}$;

$x=\left(2 S_{2} / \Delta t+Q_{2}\right)$, calculado na etapa 4 ;

$x_{1}$ e $x_{2}=(2 S / \Delta t+Q)$, da etapa 2 , no tempo 1 e 2 ;

$y_{1}$ e $y_{2}=$ vazões de descarga total, da etapa 2 , no tempo 1 e 2 .

6) Calcula-se (2S2/ $\Delta \mathrm{t}-\mathrm{Q} 2)$ subtraindo $2 \mathrm{Q} 2$ de $(2 \mathrm{~S} 2 / \Delta \mathrm{t}+\mathrm{Q} 2)$, em seguida retorna-se para a etapa 3. Sendo que, $(2 \mathrm{~S} 2 / \Delta \mathrm{t}-\mathrm{Q} 2)$ em qualquer tempo será $(2 \mathrm{~S} 1 / \Delta \mathrm{t}-\mathrm{Q} 1)$ para o tempo seguinte.

7) Repete-se as etapas anteriores até gerar-se a hidrógrafa defluente do reservatório.

Por fim, foi determinada a eficiência de amortecimento do reservatório (Equação 21) (HidrostudioFCTH, 2013).

Onde:

$$
E=\left[\left(Q p_{e n t}-Q p_{\text {sai }}\right) / Q p_{\text {ent }}\right] \times 100
$$

$E=$ eficiência (\%);

$Q p_{\text {ent }}=$ vazão de pico do hidrograma de entrada do reservatório $\left(\mathrm{m}^{3} / \mathrm{s}\right)$;

$Q p_{\text {sai }}=$ vazão de pico do hidrograma de saída do reservatório $\left(\mathrm{m}^{3} / \mathrm{s}\right)$.

\section{Resultados e Discussões}

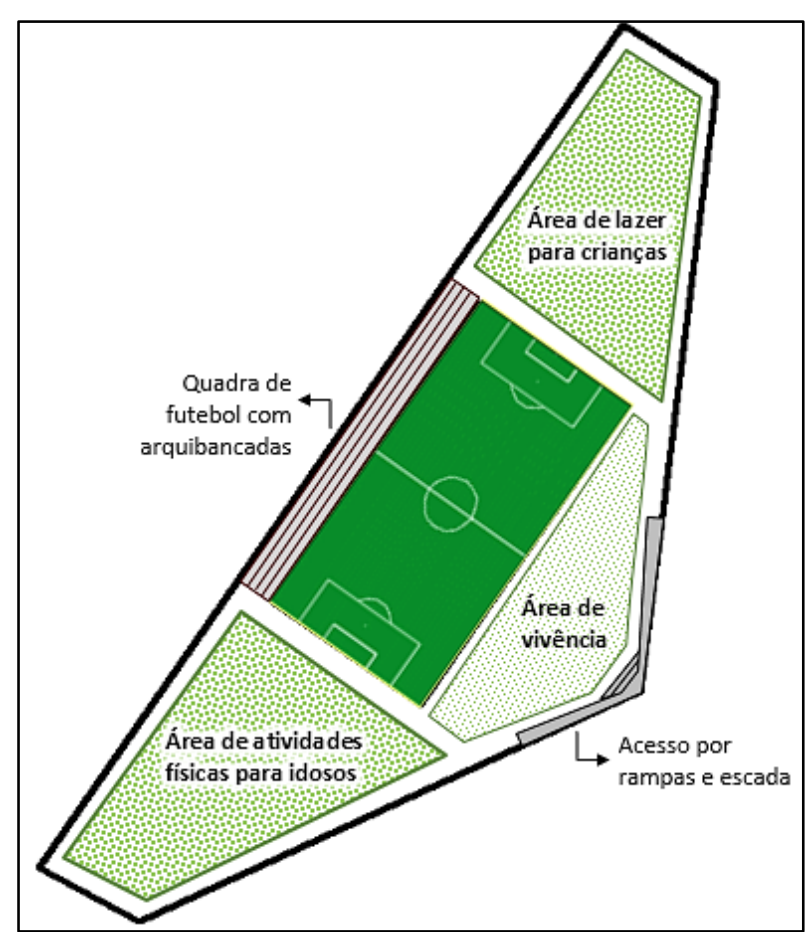

0 reservatório $R$, foi dimensionado para tempos de recorrência (TR) de 5, 10 e 25 anos, de modo a avaliar-se suas respectivas eficiências de controle de enchentes.

A fim de idealizar a adaptação do reservatório para uso dos moradores da região, este foi definido como uma bacia de detenção off-line aberta, com operação por gravidade. Propõe-se um projeto, como ilustra a Figura 6, com áreas de vivência, de lazer para crianças e de exercícios para idosos, além de uma quadra de futebol de grama. Vale ressaltar a intenção de se manter o mínimo possível de áreas impermeáveis no reservatório, de forma a favorecer a infiltração das águas pluviais.

Figura 6. Arranjo do projeto paisagístico para o reservatório. 
Conforme a metodologia apresentada, o tempo de concentração na bacia foi estimado em cerca de 0,34 horas, ou 20,6 minutos. Os cálculos resultaram ainda em um índice $C N$ ponderado equivalente a 82 , e um armazenamento máximo de $55,6 \mathrm{~mm}$. Com isso, foi possível determinar o hidrograma unitário triangular (HUT) da bacia do reservatório $R$ (Figura 7), e, para os TR de 5, 10 e 25 anos, a precipitação excedente na bacia (Figura 8) e hidrogramas de projetos correspondentes (Figura 9). A vazão de pico obtida para os TR de 5, 10 e 25 anos foram, nesta ordem, iguais a $38,78 \mathrm{~m}^{3} / \mathrm{s}, 50,15 \mathrm{~m}^{3} / \mathrm{s}$ e $68,82 \mathrm{~m}^{3} / \mathrm{s}$, gerando um volume total de escoamento superficial de $51.940 \mathrm{~m}^{3}, 67.345 \mathrm{~m}^{3}$ e $92.740 \mathrm{~m}^{3}$, respectivamente.

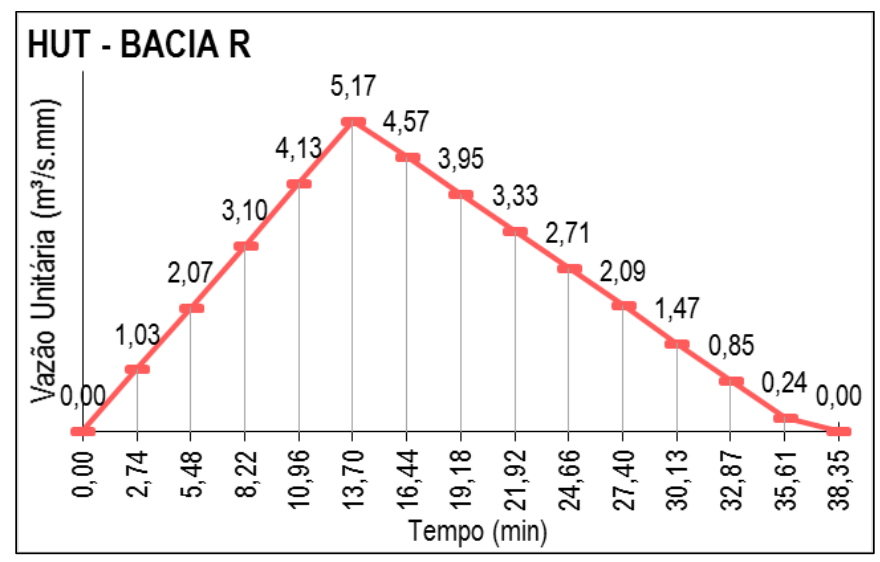

Figura 7. Hidrograma Unitário Triangular da bacia do reservatório.

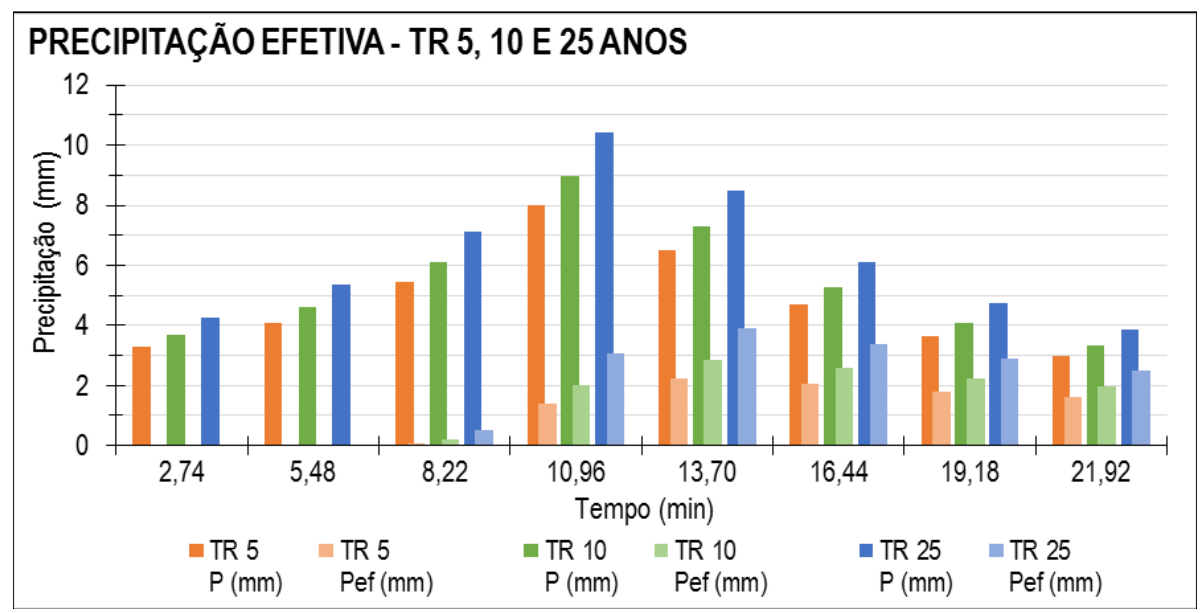

Figura 8. Precipitação excedente na bacia do reservatório, para TR de 5, 10 e 25 anos.

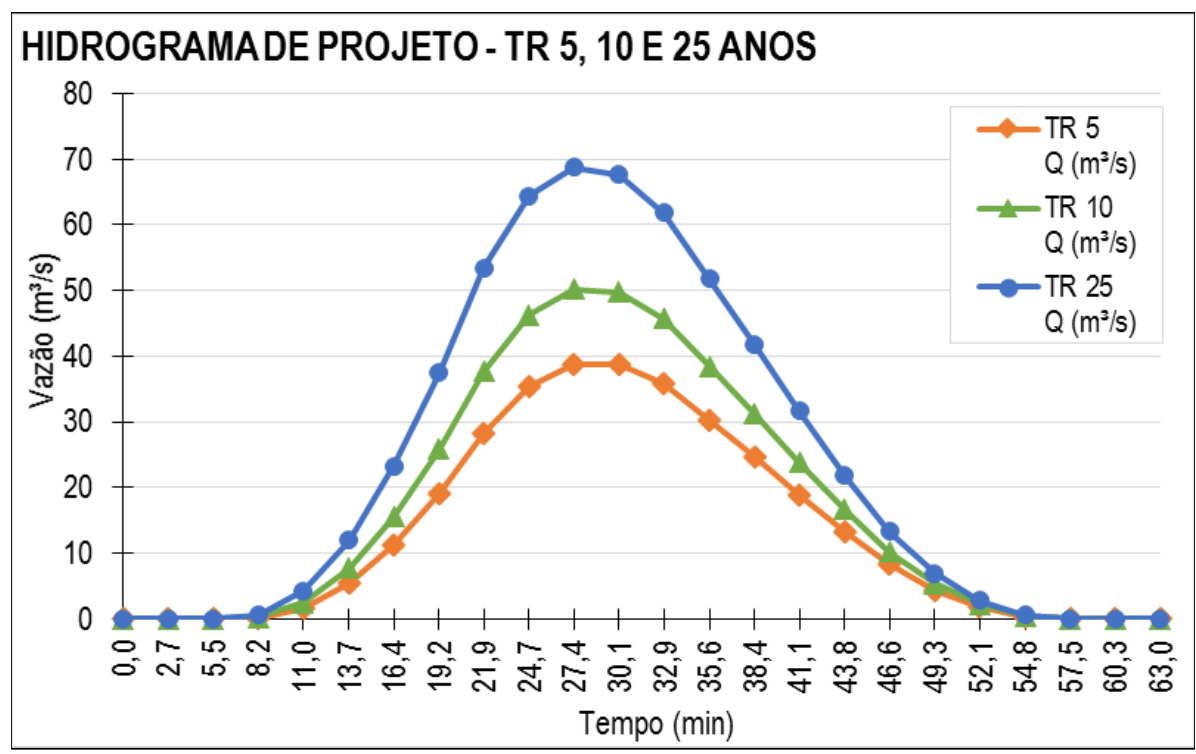

Figura 9. Hidrograma de projeto da bacia do reservatório, para TR de 5, 10 e 25 anos. 
Seguindo os procedimentos de cálculo do Método Modificado de Puls, foi determinada a curva cota $x$ volume do reservatório $R$, assim como as curvas cota $x$ vazão de descarga, correspondentes aos TR de 5,10 e 25 anos. Para cada TR avaliado, as dimensões do vertedor e orifício foram definidas de forma a propiciar uma maior eficiência de amortecimento do reservatório $R$, conforme a Tabela 4 .

Por fim, os routings do reservatório $\mathrm{R}$ foram estimados para os TR de 5, 10 e 25 anos (Figura 10), nos quais as vazões de pico foram reduzidas, de $38,78 \mathrm{~m}^{3} / \mathrm{s}$ para $5,22 \mathrm{~m}^{3} / \mathrm{s}, 50,15 \mathrm{~m}^{3} / \mathrm{s}$ para $14,48 \mathrm{~m}^{3} / \mathrm{s}$ e de $68,82 \mathrm{~m}^{3} / \mathrm{s}$ para $31,95 \mathrm{~m}^{3} / \mathrm{s}$, respectivamente. Isto corresponde a uma eficiência de amortecimento de $86,5 \%, 71,1 \%$ e $53,6 \%$, na mesma ordem. 0 tempo de retardo das vazões de picos foi de 16,46 min para o TR de 5 anos, 13,70 min para 10 anos e 11 min para 25 anos. Isto indica que, o reservatório seria capaz de reduzir as dimensões e danos de inundações, de forma mais relevante, para eventos mais frequentes de cheia.

Tabela 4. Dimensões do vertedor e orifício do reservatório, para TR de 5,10 e 25 anos.

\begin{tabular}{|c|c|ccc|}
\hline \multicolumn{2}{|c}{ Dimensões } & TR 5 & TR 10 & TR 25 \\
\hline \multirow{3}{*}{ Vertedor } & Lb (m) & 1,0 & 1,5 & 2,0 \\
\cline { 2 - 5 } & hlam $(\mathrm{m})$ & 1,0 & 1,0 & 0,5 \\
\hline \multirow{3}{*}{ Orifício } & Lo (m) & 1,0 & 3,0 & 8,1 \\
\cline { 2 - 5 } & Ho (m) & 1,0 & 1,0 & 1,0 \\
\hline
\end{tabular}

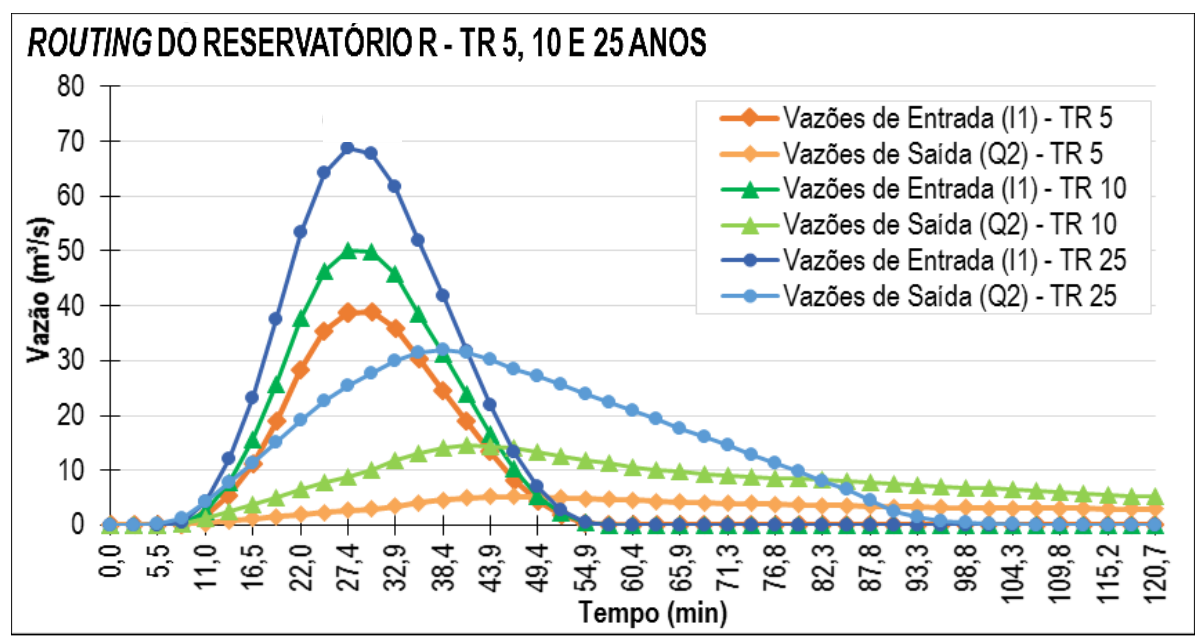

Figura 10. Routing do reservatório, para TR de 5, 10 e 25 anos.

Vale ressaltar, que seria possível a construção de um reservatório de detenção com profundidades maiores do que a proposta, a fim de aumentar a eficiência de amortecimento da cheia. No entanto, seria necessário avaliar a viabilidade técnica e econômica das alternativas, uma vez que profundidades maiores do que as do sistema de drenagem do local, exigem a construção de tubulações de saída mais extensas ou o uso de bombas para o esvaziamento do reservatório.

Devem ainda ser consideradas ações e intervenções propostas no âmbito do Plano Diretor de Manejo de Águas Pluviais (PDMAP) da bacia de estudo, referentes à reservação em outros pontos, dragagem dos trechos de córregos urbanos assoreados, recuperação de margens degradadas, além de medidas não-estruturais de forma complementar.

\section{Conclusão}

A proposta principal do presente trabalho foi dimensionar uma bacia de detenção off-line aberta, com operação por gravidade, para amortecimento de enchentes com tempo de recorrência de 5, 10 e 25 anos na bacia hidrográfica do rio Jacaré. 0 reservatório proposto estaria localizado próximo à Rua Dois de Maio, e possuiria uma área total de $16.000 \mathrm{~m}^{2}$ e profundidade de $3 \mathrm{~m}$, com capacidade de armazenamento de $48.000 \mathrm{~m}^{3}$. 
De acordo com os resultados, o reservatório teria então, uma eficiência de amortecimento de cerca de $86 \%$ para chuvas com um tempo de recorrência de 5 anos, $71 \%$ para 10 anos e $54 \%$ para 25 anos.

Por estar localizado em uma região residencial, foi proposta a adequação da bacia de detenção em uma quadra de futebol de grama, além de uma área de vivência, um espaço de lazer para crianças e de exercícios para idosos. Desta forma, seria possível agregar valor social à comunidade local.

Por fim, recomenda-se que sejam consideradas intervenções propostas no Plano Diretor de Manejo de Águas Pluviais (PDMAP) da bacia de estudo, bem como a elaboração de estudos mais detalhados, para avaliação da viabilidade técnica e econômica do reservatório proposto.

\section{Referências}

Akan, A. O. (1993). Urban Stormwater Hydrology: A Guide to Engineering Calculations. Lancaster [Pennsylvania]: Technomic.

Arnfield, A. J. (2003). Review. Two decades of urban climate research: a review of turbulence, exchanges of energy and water, and the urban heat island. International Journal Of Climatology (23), 1-26.

Bragança, L. S. (2015). Manejo de águas pluviais urbana : determinação do hidrograma de projeto da bacia do Rio Jacaré, zona norte da cidade do Rio de Janeiro. Trabalho de Conclusão de Curso I (Graduação em Engenharia Ambiental). Universidade Federal Fluminense. Niterói, RJ, Brasil.

Canholi, A. P. (2005). Drenagem Urbana e Controle de Enchentes. São Paulo: Oficina de Textos.

Cheng, C. K., \& Chan, J. C. (2012). Impacts of land use changes and synoptic forcing on the seasonal climate over the Pearl River Delta of China. Atmospheric Environment(60), 25-36.

EMBRAPA. (2004). Mapeamento pedológico e interpretações úteis ao planejamento ambiental do Município do Rio de Janeiro. Rio de Janeiro: Embrapa [Empresa Brasileira de Pesquisa Agropecuária].

FCTH. (2012a). Manual de Drenagem e Manejo de Águas Pluviais. Aspectos Tecnológicos: Fundamentos. Volume II. São Paulo: Fundação Centro Tecnológico de Hidráulica (FCTH). Secretaria Municipal de Desenvolvimento Urbano (SMDU).

FCTH. (2012b). Manual de Drenagem e Manejo de Águas Pluviais. Aspectos Tecnológicos: Diretrizes para projetos. Volume III. São Paulo: Fundação Centro Tecnológico de Hidráulica (FCTH), Secretaria Municipal de Desenvolvimento Urbano (SMDU).

Güneralp, B., Güneralp, I., \& Liu, Y. (Fevereiro de 2015). Changing global patterns of urban exposure to flood and drought hazards. Global Environmental Change, 31, 217-225.

Guo, X., Fu, D., \& Wang, J. (2006). Mesoscale convective precipitation system modified by urbanization in Beijing City. Atmospheric Research (82), 112-126.

Hidrostudio-FCTH. (2013). Plano Diretor de Manejo de Águas Pluviais da cidade do Rio de Janeiro - Bacia Hidrográfica do Canal do Cunha. Rio de Janeiro: Consórcio Hidrostudio Engenharia - Fundação Centro Tecnológico de Hidráulica. Fundação Rio Águas — Prefeitura da Cidade do Rio de Janeiro.

IBGE. (2014). Downloads Geociências. Acesso em 10 de Junho de 2015, disponível em IBGE - Instituto Brasileiro de Geografia e Estatística: http://downloads.ibge.gov.br/downloads_geociencias.htm

Kazmierczak, A., \& Cavan, G. (Setembro de 2011). Surface water flooding risk to urban communities: Analysis of vulnerability, hazard and exposure. Landscape and Urban Planning, 103, 185-197.

Oke, T. R. (1987). Boundary Layer Climates. (2nd ed.). Taylor \& Francis Group. 
Porto, R. L. (1995). Escoamento Superficial Direto. Em C. E. Tucci, R. L. Porto, \& M. T. Barros, Drenagem Urbana (pp. 107-165). Porto Alegre: Editora da UFRGS/ABRH.

Rio Águas. (2010). Instruções Técnicas para Elaboração de Estudos Hidrológicos e Dimensionamento Hidráulico de Sistemas de Drenagem Urbana. Rio de Janeiro: Subsecretaria de gestão de bacias hidrográficas - Rio Águas, Secretaria Municipal de Obras, Prefeitura da Cidade do Rio de Janeiro.

SCS. (junho de 1986). Urban hydrology for small watersheds. SCS - SOIL CONSERVATION SERVICE. U.S. Department of Agriculture, Washington, DC.

Seto, K. C., \& Shepherd, J. M. (Agosto de 2009). Global urban land-use trends and climate impacts. Current Opinion in Environmental Sustainability, 1, 89-95.

Strelkoff, T. (1980). Modified Puls routing in Chuquatonchee Creek. California: US Army Corps of Engineers Hydrologic Engineering Center. DACW05-80-P-0324.

Taylor, J., Lai, K. M., Davies, M., Clifton, D., Ridley, I., \& Biddulph, P. (2011, April). Flood management: Prediction of microbial contamination in large-scale floods in urban environments. Environment International, 37, 1019-1029.

Tomaz, P. (2002). Cálculos Hidrológicos e Hidráulicos para Obras Municipais. São Paulo: Editora Navegar.

Tucci, C. E. (2005). Gestão de Águas Pluviais Urbanas. Ministério das Cidades. Global Water Partnership. World Bank. Unesco.

Tucci, C. M. (2007). Inundações Urbanas (Vol. 11). (C. A. V., Ed.) Porto Alegre: Rhama.

Tucci, C. M. (2009). Hidrologia, ciência e aplicação. (4a ed.). Porto Alegre: Editora da UFRGS/ABRH.

Yang, L., Tian, F., \& Niyogi, D. (Março de 2015). A need to revisit hydrologic responses to urbanization by incorporating the feedback on spatial rainfall patterns. Urban Climate, 12, 128-140. 